\title{
Cultural Competent Care in Hong Kong
}

\author{
Baljit Kaur
}

\begin{abstract}
Hong Kong is an international city with a mixture of various cultures. It is one of the most densely populated areas in the world with a population of seven million people; however, they are not just Hong Kong people. Although 92\% of population is Chinese Hong Kong people, there are almost sixty thousands people come from other countries. With different backgrounds, histories, and habits from different cultures, there are different responses and beliefs to illness, treatment and nursing care. In order to prevent conflict between cultures and provide the most suitable and effective care to patients, culturally competent care should be promoted. Different suitable strategies for nurses to promote culturally competent care in Hong Kong is essential such as establishing cultural competent in clinical setting, education, self learning materials and systematic information collection.
\end{abstract}

\section{Index Terms-Culture, competent care, Hong Kong.}

\section{INTRODUCTION}

Culture is traditional ideas, which are learned, shared and passed knowledge of values, beliefs, and life ways from one generation to the next generation [1]. In promoting cultural competence, Donini-Lenhoff, \& Hendrick concluded that culture should include thoughts, styles of communication, ways of integrating, views on roles and relationships, values, practices and custom [2].

Cultural diversity refers to differences in culture. It may cause by differences in race, ethnicity, national origin, religion, age, gender, sexual orientation, ability or disability, social and economic status or class, education, and related attributes of groups of people in society [3].

Culturally competent care means providing respectful care that is consistent with cultural health beliefs of the client and family members [3]. It is providing care with overcoming the conflict derived by cultural diversity between the client, family and care provider. Campinha-Bacote specified the process of cultural competence in the delivery of healthcare service, is an ongoing process in which the health professional continuously strives to seek the ability and availability to work effectively within the cultural context of the client. And the concept of cultural competence includes 5 aspects, "cultural desires", "cultural awareness", "cultural knowledge", "cultural skill", and "cultural encounters" [4].

Transcultural nursing is a specialty wording within nursing, focused on the comparative study and analysis of different cultures and subcultures [2]. Transcultural nursing requires sophisticated assessment and analytic skills and the ability to plan, design, implement and evaluate nursing care for individuals, families, groups and communities representing

Manuscript received July 22, 2014; revised October 20, 2014.

Baljit Kaur is with The University of Hong Kong, Indian (e-mail: Baljit@connect.hku.hk). various cultures [5]. The goal of transcultural nursing is to provide "cultural universal nursing care" and "cultural specific nursing care" for the health and well being for client in facing cultural diversity. "Cultural universal" is commonly shared values, norms of behaviors that are similarly held among cultures [6]; and "cultural specific" is particular values, belief and pattern of behavior of a unique group that do not tend to be shared with members of other cultures [3].

\section{IMPORTANCE OF CULTURAlly COMPETENT CARE}

Promoting culturally competent care brings numerous benefits to patients, nurses and the nursing care. This shows the importance of culturally competent care. When nurses provide culturally competent care, they need well-rounded knowledge. Through promotion of culturally competent care such as by the mean of education, it enriches the knowledge of the health care professionals [7], [8]. It also helps improving the quality of care, decreasing inappropriate differences in the characteristics and closing the gaps in health status across diverse populations [2], [8]. The reason is that nurses are more sensitive to the cultural needs of patients and the interventions would become specific and accurate to patients [8]. With increasing understanding of different cultures, providing care will be more effective with better allocation of resources. Therefore, it will maximize the use of limited resources [7].

Improve culturally competence of nurses would also increase the patients understanding of and adherence to treatment recommendation, help developing therapeutic relationship between clients and nurses and increase patient's satisfaction [7]-[9].

\section{Culturally Competent Care IN Hong Kong}

Hong Kong is an international city which consists of multiple races. Most of the population in Hong Kong is Chinese; however, more than sixty thousands Hong Kong citizens came from other nations, e.g. Indonesia, Philippine, Britain and India. Regarding to the difference in nationality, cultural diversity may exists due to different races, ethnicities, national origins, religions, educational levels. Owing to the differences, people would have different beliefs and behaviors towards health and illness so care providers may find it difficult when providing care to them. In fact, some actions have been already taken for promoting cultural competent care in Hong Kong.

Nursing education in Hong Kong, had already engaged and trained frontline staff to promote equal opportunities to different nationality [10], and hence, this promote transcultural nursing. As education is a direct way to transfer knowledge from one to another, it can arises the awareness of 
cultural diversity and stress the importance of culturalcompetent care of nurses [11], which may enhance new generation of nurses to provide culturally competent care granted by professional knowledge.

\section{Cultural Assessment}

Cultural assessment can help nurses to identify cultural differences of the clients. There are two famous models for cultural assessment: One is Leininger's Sunrise Model and another is Giger and Davidhizar's Transcultural Assessment Model. They provide framework and guidance for assessment in order to provide culturally competent care and help the development, test and organize the emerging body of knowledge in transcultural nursing.

\section{A. Leininger's Sunrise Model}

Leininger's Sunrise Model [6] showed that factors include worldwide, social structure, language, ethno history, environmental context, and the generic (folk) and professional systems are influencing cultural care. And those factors are recognized as major influences on human expressions and experiences related to health, illness, and well-being or on facing disabilities or death.

\section{B. Giger and Davidhizar's Transcultural Assessment Model}

Meanwhile, Giger \& Davidhizar believed that each client is culturally unique individual and clients should be assessed according to 6 phenomena: environmental control, biological variations, social organization, communication, space and time [5].

\section{1) Environmental control}

Firstly, environmental control refers to the ability of particular cultural group members to plan activities to control nature and direct environmental factors [5]. It is related to individual's belief in internal control and external control. According to Giger \& davidhizar, if an individual has less belief in internal control and more in external control, he may have fatalistic view and think seeking health care is useless [12]. Traditional health and illness beliefs in Hong Kong help to illustrate this cultural phenomenon. Traditional Chinese medicines consist of techniques such as cupping, acupuncture, Qigong, Tai Chi, herbal medicines, i.e. making herbal tea or soup to 'reduce heat', which is a mean to clear dampness or as a tonic [13]. These health practices are common in Hong Kong. Additionally, a research study was conducted to assess the health care consumption pattern in Hong Kong [14]. The result revealed besides visiting western allopathic medical practitioners, about $10 \%$ of respondents visited traditional Chinese medical practitioners [14]. Also, research studies conducted in Hong Kong by Lam, Chung and colleagues showed respondents considered traditional Chinese medicines are being better in curing the root of the problem and $36.9 \%$ of the respondents reported self use of Chinese herbal medicine respectively [15], [16]. Therefore, it is believed that there are a certain proportion of Hong Kong residents who believe in traditional Chinese medicines.

\section{2) Biological variations}

Secondly, biological variations mean the several ways in which people from one cultural group differ biologically from members of other cultural groups such as body build and structure, skin color, genetic variations, susceptibility to disease and nutritional variations [5]. It is a starting point to recognize the biological difference among various cultural and racial groups in order to provide culturally competent care [12]. In Hong Kong, there are various racial groups that have different skin colors, genetic variations, etc. According to the Census and Statistics Department, apart from Chinese, $1.9 \%$ of the total population of Hong Kong is other ethnicities such as Indonesian, Filipino, White, Indian and Pakistani [16], [17]. The understanding of biological variations between them helps to provide more appropriate nursing care to that specific racial group in Hong Kong. For example, the Chinese are more sensitive to the cardiovascular effects of Propranolol than the White [15].

\section{3) Social organization}

Thirdly, social organization refers to the family unit and the social group organizations which patients and families may identify, such as religions and family structure [5]. According to Information Services Department, there is a variety of religious groups in Hong Kong including Buddhism, Taoism, Confucianism, Christianity, Islam, Hinduism, Sikhism and Judaism [18]. By understanding the difference in health beliefs and practices among various religious groups in Hong Kong, nurses can provide care more effectively without breaking taboos.

\section{4) Communication}

Fourthly, communication is a continuous process by which one person may affect another through written or oral language, gestures, facial expressions, body language, space, or other symbols. It includes language differences, verbal and nonverbal behaviors and silence. Culture is transmitted and preserved through communication. It is the most important obstacles in providing multicultural health care and all stages of the patient-caregiver relationship are affected by communication [5]. In Hong Kong, $88 \%$ of people speak Cantonese as their dialect. Other Chinese dialects, for example, Hakka and Teochiu are also presented. Moreover, since the reunification in 1997, Mandarin becomes more widely spoken in Hong Kong.

\section{5) Space}

Fifthly, space refers to the distance between individuals when they interact. Personal spaces refer to people's behaviors and attitudes toward the space around themselves. With different cultures, rules concerning personal distance vary. According to Engleberg \& Wynn, there are four different zones of interpersonal space: intimate distance, personal distance, social distance and public distance [18]. Intimate distance ranges from touching to about $46 \mathrm{~cm}$ apart, it reserves for lovers, children, family members and friends. Personal distance begins about an arm's length away. This space is commonly used in conversations with friends and in group discussions. Social distance ranges from $1.2 \mathrm{~m}$ to $2.4 \mathrm{~m}$ away from the person and is reserved for strangers and newly formed groups. Public distance includes anything more than $2.4 \mathrm{~m}$ away, it reserves for large audiences and is used for speeches, lectures, and theater. 


\section{6) Time}

Sixthly, time is important in the interpersonal communication. Among different cultural groups, the viewing of time in the present, past or future varies. For instance, preventive actions are motivated by a future reward, so preventive health care requires some future time orientation. Future-oriented people are concerned with long range goals and measures in the present to prevent illness in the future. Time is important in health-care measures, for long-term planning and explanations of medications schedules. In Hong Kong, some people are future-oriented as they concern measures to prevent illness in the future. An example is from a report of Lam, Chan, Ho \& Chan showed that, $80 \%$ Hong Kong citizens realized that exercise is a way to prevent heart disease [19].

\section{Strategies to Promote Culturally Competent CARE IN HONG KONG}

In addition to the actions which have been taken, various strategies have to be implemented to promote culturally competent care in Hong Kong. These strategies include establishing culturally competent care in clinical setting, education, self-learning, and systemically information collection.

\section{A. Establish Cultural Competent Care in Clinical Setting}

Establishing culturally competent care in clinical setting is one of the strategies. Nurses would participate in setting protocol for guidance of their daily routine and designing assessment form based on cultural assessment models. These ways may ease nurses in providing suitable care for patients with different cultural backgrounds. Moreover, in order to communicate effectively with the patients, nurses may help recruiting and retaining diverse employees and co-operate with them and nurses should make use of interpreter services or bilingual providers [8], [20]. Furthermore, senior nurses in clinical setting should act as role models and give support to other allied health staff for providing culturally competent care [3].

\section{B. Education}

Education is another important strategy to promote culturally competent care [20], [21], [23]. A research showed that education can increase nurses' cultural competence, no matter in their cultural knowledge, sensitivity and skills [24]. Many other literatures have also recommended offering cultural competence training for health care institutions, nursing administrators, and nursing educators [8], [25], [26].

In order to increase nurses' cultural knowledge, sensitivity and skills, lectures and sharing by experts or nurses who are familiar with other cultural backgrounds can be provided to nurses who are lack of the related cultures' knowledge. For example, there was a cooperation and exchange program on transcultural nursing leadership between United States and China, Wenzhou, they shared professional experiences in nursing care and facilitated nurses on joint research, publications and education [26]. In addition, there are many other means of education that can increase nurses' cultural competence, such as, case study, group discussion, experience sharing, role play and play related video [21], [25], [27 ].

Through education, nurses would learn about cultural competent theories and skills (e.g. Giger and Davidhizar's Transcultural Assessment Model/Leininger's sunrise model). And they would know more about cultural groups they would contact the most. In Hong Kong real situation, it would be Indonesian or Filipino, as they are the largest proportion of foreign people in Hong Kong. Education not only could enrich nurses' knowledge of the history of different cultural groups, and ethnic variation in values, beliefs and ways of life of the community, but also the differences related to religion, gender, food preferences and other related cultural differences [3], [8], [22], [25].

Appropriate verbal and nonverbal communication patterns within the group can be educated in order to have better communication with people from different cultures and reduce risk of miscommunication or misunderstanding [8]. Nurses could learn and consider the appropriate ways to cope with the cultural differences after they have more understanding of different cultural beliefs and preferences. Meanwhile, education could help raising motivation on providing culturally competent care and respect to different culture, and help providing emotional support, physical comfort and adequate patient teaching [8], [21], [26].

\section{Self-Learning}

Thirdly, enhancing nurses' self-learning ability and motivation is one of the strategies to promote culturally competent care. Perng and colleagues stated that nurses have their own ability to learn continuously in order to make their knowledge base up to date [21]. Therefore, nurses have the responsibility to learn about culturally competent care. Nurses may learn about different cultures through enhancing their self-learning by spending time within the community and attending local events. They can also surf the web, read literatures and researches and seek the community's input and feedback for the health program which may help their interventions to incorporate the traditional values, beliefs and ways of life [22]. Furthermore, they can actively exchange and share their own learning process and results with other nurses [21].

\section{Systematic Information Collection}

Lastly, a well systematic information collection can help nurses to promote culturally competent care in Hong Kong. Three types of information would help nurses to promote culturally competent care [20]. Firstly, nurses can collect and utilize accurate demographic, cultural, epidemiological and clinical outcome data for racial and ethnic groups. Then, they can record the complaints by different cultural background patients and staffs about unfair culturally insensitive or discriminatory treatment, or even difficulties in accessing services or denial of services. Base on that information, nurses may find out different ethnical or cultural needs and also, they can locate resources and assets of the surrounding community to provide hints for implementing culturally competent care which are most effective. Finally, nurses can refer to annual progress report which has documenting progress in implementing the culturally competent care, no matter the information about programs or even staffing and 
resources

\section{DISCUSSION}

Although it is important to promote the culturally competent care and we can benefit from it, there are some barriers among nurses and organization which hinder the promotion and implementation of culturally competent care in Hong Kong.

It is complicated for nurses to learn various cultures within a short period of time. Owing to the fact that Hong Kong is a cosmopolitan city with different cultures, there are more than 10 other races in Hong Kong [17]. Each person from different cultures has his or her own set of health beliefs and practices that are unique, that makes a difficult task for nurses to take patient's cultural beliefs and values into account and to meet the needs of culturally diverse patients in providing quality care.

Nurses working in Hong Kong have to take care of large number of patients. There is a great short of nurses and Hospital Authority admits each nurse cared for 10 to 12 patients on average while the global standard is only pegged at four to six [28], [29]. Such heavy workload occupied most of their duty time and they may think learning and considering cultural differences is time consuming as it is less important than other duty tasks.

Although health organization may have culturally competent care training for nurses, most of the nurses just barely touch the idea of culturally competent care. They would actually lack incentive and motivation to have continuous learning which certainly hinders the promotion of culturally competent care.

Nurses who have personal biases and lack of openness are difficult to accept other cultures as they have their own preference and tendency. Also, they have long been using their own usual practice in providing care for the local communities. Thus, nurses would not be open-minded to other cultures even they have recognized the significance of culturally competent care. And even though they have already recognized the importance of promoting culturally competent care, it is difficult for them to change or they resist changing their usual practice in providing culturally and linguistically appropriate care to culturally diverse patients. It is because change in practice may make the procedure more complicated and lengthen the time needed.

There is inadequate financial resource in providing culturally competent care training and the health organization is reluctant to expand resources for cultural learning, these make nurses feel frustrated [30]. With inadequate resources on training, Hong Kong nurses can rarely increase the awareness of racial and ethnic disparities in health care. As a result, nurses cannot improve the quality of care and cultural and linguistic barriers which they may encounter in clinical setting cannot be eliminated.

In addition, health organization as a facilitator of promoting culturally competent care failed to motivate or reward nurses for cultural studies and there is shortage of role models for promoting culturally competent care. Even though some related training are provided, most of nurses are not wholeheartedly to learn for having better communicationand understanding of one's cultural background, health beliefs and values in providing quality care.

\section{CONCLUSION}

To nurses, it is recommended to be open-minded which focuses on the attitude and personality of the nurses. It is essential for nurses to be open-minded when facing foreign patients because they have totally different cultures and customs from Hong Kong. It is suggested nurses that getting along with the patients who have different cultures and customs without prejudice. It is not an easy task to make a decision without any prejudice to the foreign patients, however, it is necessary to have a try and it would be a start of success.

From a qualitative research study, the participated nurses stated that opening their hearts and minds may help them increasing understanding of other cultures and peoples, increasing understanding of global sociopolitical and health issues, increasing one's commitment to make a difference, experiencing personal and professional growth, contributing to professional development in the host country, making interpersonal connections and developing cultural competence [31].

In addition, nurses should remember their responsibility of gaining knowledge and skills that would results better quality of care. It is always true that well preparation is a critical factor for success; this is not except the implementation of culturally competent care.

To organization, it is recommended to allocate more resources to develop culturally competent care [21]. For example, expand resources for launching culturally competent care courses and training, conducting researches, and provide rewards. Undoubtedly, allocating resources effectively and efficiently is a key to success in promoting culturally competent care.

In addition, it is suggested to show positive results on promoting culturally competent care from other countries which may increase the nurses' motivation and alert the need of implementation in health care setting. Apparently, there are many related successful case. In Taiwan, the researchers successfully provided educational programme for nurses that enhanced nurses' cultural competence [21] and professional knowledge and skills related to transcultural nursing can be improved by the exchange between China and United States [26].

Added to the above, as heavy workload and great work stress are the challenges for nurses to attend the culturally competent care training, it is suggested to provide incentive to increase the motivation of self-learning [3], [20]. For instance, the organization can provide allowance for the nurse who attends the cultural competent care training. Also, organization can include the course of culturally competent care in the career training program to ensure all nurses have a basic idea of what is culturally competent care. Moreover, it is recommended to encourage nurses to attend training by giving those rewards such as certification or medal.

There is a possibility for nurses to contact with patients who come from other cultures who may have different 
perception to the nursing care because of their different cultural backgrounds. Culturally competence is an efficient process for nurses to seek the ability and availability to work effectively and respectfully within the cultural context of the patients. Therefore, culturally competent care is increasingly important in Hong Kong, an international city.

For successfully applying culturally competent care in Hong Kong, proper and precise assessment is essential to do so. Transcultural assessment model is a suitable way for nurses to do the assessment to patients who have different cultural backgrounds. The main advantages of culturally competent care are it could impact the patient with the positive satisfaction, maximize the using of limited resources in hospital, meet the patient's need and improve the quality of care. Although several benefits in promoting culturally competent care were noted, accessibilities for nurses to implement in clinical setting are not that easy. Heavy workload, lack of motivation and resistance to change are the main barriers for nurses to implement during work. However, through education, self-learning and information collection, nurses will have better understanding on providing cultural competent care when encountering patients with different cultures in Hong Kong. In addition, nurses should be open-minded to acquire knowledge and skills needed to assess and care for patients that may encounter in their career. Therefore, culturally competent care is an effective platform for nurses to implement high quality and effective nursing care to patients from different cultures.

\section{REFERENCES}

[1] P. R. Rose, Cultural Competency for Health Administration and Public Health, Massachusetts: Jones and Bartlett Publishers, 2011, ch. 2, pp. 45-47.

[2] F. G. D. Lenhoff and H. L. Hendrick, "Increasing awareness and implementation of cultural competence principles in health professions education," Journal of Allied Health, vol. 29, no. 4, pp. 241-245.

[3] M. M. Andrews and J. S. Boyle, Transcultural Concepts in Nursing Care, $5^{\text {th }}$ ed., Philadelphia: Lippincott Williams \& Wilkins, 2008, pp. 23-28.

[4] J. C. Bactoe, The Process of Cultural Competence in the Delivery of Health Care Services, $4^{\text {th }}$ ed., Cincinnati, OH: Transcultural C.A.R.E. Associates, 2008, pp. 13-48.

[5] J. N. Giger and R. E. Davidhizar, Transcultural Nursing, 4th ed., Missouri: Mosby, 2004, pp. 68-72.

[6] M. M. Leininger and M. R. McFarland, Transcultural Nursing: Concepts, Theories and Practices, New York: McGrawHill, 2002, pp. 28-36.

[7] R. Alexander, "Diversity, Cultural Competence and nursing student" Nsna Imprint, vol. 2, no. 1, pp. 43-45, 2002.

[8] Nursing Council of Hong Kong, Code of Professional Conduct and Code of Ethics for Nurses in Hong Kong, 2002.

[9] The Centre for Effective Collaboration and Practice, How is Cultural Competence Integrated in Education, 2001.

[10] J. N. Giger and R. E. Davidhizar, "The Giger and Davidhizar transcultural assessment model," Journal of Transcultural Nursing, vol. 13, no. 3, pp. 185-188, 2002.
[11] N. Robinson, "Integrated traditional Chinese medicine," Complementary Therapies in Clinical Practice, vol. 12, no. 2, pp. 132-140, 2006.

[12] G. M. Leung, I. O. Wong, W. S. Chan, S. Choi, and S. V. Lo, "The ecology of health care in Hong Kong," Social Science and Medicine, vol. 61, no. 3, pp. 577-590, 2005.

[13] T. P. Lam, "Strengths and weaknesses of traditional Chinese medicine and western medicine in the eyes of some Hong Kong Chinese," Journal of Epidemiology and Community Health, vol. 55, no. 10, pp. 762-765, 2001

[14] V. C. Chung, S. Hillier, C. H. Lau, S. Y. Wong, E. K. Yeoh, and S. M. Griffiths, "Referral to and attitude towards traditional Chinese medicine amongst western medical doctors in postcolonial Hong Kong," Social Science and Medicine, vol. 72, no. 2, pp. 247-255, 2011.

[15] Census and Statistics Department, 2011 Hong Kong Population Census, 2013.

[16] I. N. Engleberg and D. R. Wynn, "Working in groups: communication principles and strategies," My Communication Kit Series, 4th ed., Maryland: Allyn \& Bacon, 2006.

[17] T. H. Lam, B. Chan, S. Y. Ho, and S. K. Chan, Report on Healthy Living Survey 1999, Hong Kong: Department of Health, 1999.

[18] U.S. Department of Health and Human Services of Minority Health, National Standards on Culturally and Linguistically Appropriate Services, 2000.

[19] S. J. Perng, C. J. Lin, and J. L. Chuang, "Cultivating cultural competence through education," Tzu Chi Nursing Journal, vol. 6, no. 4, pp. 91-102, 2007.

[20] M. K. Douglas, J. U. Pierce, M. Rosenkoetter, L. C. Calliter, M. Hattar-Pollar, J. Lauderdale, J. Miller, J. Milstead, D. A. Nardi, and D. Pacquiao, "Standards of practice for culturally competent nursing care: a request for comments," Journal of Transcultural Nursing, vol. 20, no. 3, pp. 257-269, 2009.

[21] Learning nurse, Professional Development for Nurses, 2013.

[22] P. McGee, Teaching Ttranscultural Care - a Guide for Teachers of Nursing and Health Care, California: Chapman \& Hall, 1994.

[23] K. L. Capitulo, "Developing a transcultural nursing leadership institute in China," MCN American Journal Maternal Child Nursing, vol. 37, no. 5, pp. 325-330, 2012.

[24] T. B. Long, "Overview of teaching strategies for cultural competence in nursing students," Journal of Cultural Diversity, vol. 19, no. 3, pp. 102-108, 2012.

[25] M. Yeung, Nurses Charge Workload Threatens Healthcare, 2011.

[26] S. Starr and D. C. Wallace, "Self-reported cultural competence of public health nurses in a southeastern U.S. public health department," Public Health Nursing, vol. 26, no. 1, pp. 48-57, 2009.

[27] E. W. Cagape, Hong Kong Hospitals Short of 600 Nurses, 2010.

[28] S. N. Abdullah, "Towards an individualized client's care: Implication for education. The transcultural approach," Journal of Advanced Nursing, vol. 22, pp. 715-720, 1995.

[29] L. C. Callister and A. H. Cox, "Opening our hearts and minds: the meaning of international clinical nursing electives in the personal and professional lives of nurses," Nursing and Health Science, vol. 8, no. 2 , pp. 95-102, 2006.

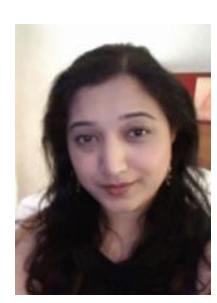

Baljit Kaur is a Hong Kong born and raised Indian She had obtained her bachelor of science (nursing) (hons) degree from Oxford Brooks University. She had obtained two master degrees. One of the master's degree is master of science (nursing) from Oxford Brooks University and the other master degree is from the University of Hong Kong in master of social science (gerontology). She is now currently a $\mathrm{PhD}$ candidate in the University of Hong Kong. Her major interest in research is transcultural nursing, nursing education, ethical and legal nursing, simulation education, primary health care and gerontology. 